\title{
Bionic arm: Mapping of elbow and wrist flexion using neural network and fuzzy logic
}

\author{
Aiswarya Lakshmi M. and Anjan Kumar Dash* \\ Department of Mechatronics Engineering, School of Mechanical Engg., SASTRA Deemed to be University, Thanjavur, India \\ * Corresponding Author : anjandash@mech.sastra.edu
}

Submitted : 20/09/2020

Revised :05/08/2020

Accepted : 12/12/2020

\begin{abstract}
Cases on limb amputation necessitate the use of Transhumeral bionic for artificial limb rehabilitation, which is controlled using Electromyographic (EMG) signals from the muscles. Before the implementation of EMG control, a mapping between the movements of an arm to the angle formed at the corresponding joints is essential to be made. Most of the works in the field of Bionics use Supervised Machine Learning models, chiefly Classification, to map muscle flexion signals to joint actuations in the bionic arm. Ample literature is also there, which uses fuzzy logic for mapping. However, there are very few literatures that compare these two methods of mapping. In this article, 2 models have been discussed regarding the mapping, and their effectiveness is compared. The first model captures elbow and wrist flexion and maps them to their respective angular displacements of joints using a fuzzy logic model. In the second model, a Pattern Recognition Artificial Neural Network (ANN) model under Supervised Machine Learning is incorporated to map elbow and wrist flexion to the corresponding joint angular displacement. The ANN is trained with elbow and wrist joint flexion values and its corresponding joint angles data, optimized, and tested in real-time. This model is verified by comparing the joint angles of a test person (measured using Goniometers) with the joint angles of Bionic models made (using a $360^{\circ}$ protractor sheet). The second model gave the insight that supervised machine learning models provide an accurate mapping to the joint flexion in the field of bionics.
\end{abstract}

Keywords: Upper limb; Flexion; Pronation; Neutral position; Supination of wrist; Neural network.

\section{INTRODUCTION}

The dexterity of a human hand is attributed to the many degrees of freedom it has, which are given by different joint movements. Handrix: Animating a Hand [1] reveals that there are 27 degrees of freedom for a human hand. The complexity of a bionic arm depends on the degrees of freedom in which it can move. Upper limb dysfunctionality is chiefly caused by amputation [2.3], limb impairment [4], Monoplegia, and Cerebral Palsy [5]. Such cases require a bionic arm for artificial limb rehabilitation. Most of the current existing Bionics use electromyographic (EMG) [6,7] methods to detect and measure muscle signals using EMG instruments and EMG sensors. In some cases, before designing a bionic arm for a patient, an Osseointegration [8,9] is done, which is a mechanical connection between the bone (where the limb is amputated) and the bionic structure. It provides higher stability and weight-bearing capacity. Targeted muscle reinnervation (TMR) is used to capture signals from the muscles at the amputated limb end to control of the bionic arm [10,11]. It was observed that the combination of TMR and electromyography techniques yielded more accurate results $[12,13]$. A bionic arm should be capable of capturing maximum movements 
such as grasping [14], flexion and extension of limbs [15] and rotation of wrist with maximum accuracy and provide the corresponding action(s).

For a bionic arm to be implemented on a patient using electromyographic [16,17] technique, it should be trained and tested with a test person ${ }^{* 1}$, which is a prerequisite stage where the bionic arm is required to replicate the actions of the test person. This stage is very crucial as the accuracy with which the bionic arm traces the path of the test person's hand can be determined and optimized $[18,19]$.

Most of the works done by pioneers in the field of prosthetics to map muscle signals to joint actuations in prosthetic arm are based on Pattern Recognition and Classification, that come under Supervised Machine Learning. Taşar, B., \& Gülten, A. (2017) used Fuzzy logic based classification method to map the forearm muscle movements to joint movements of a bionic arm, using surface electrodes [20,21,22]. Pattern recognition methods have been widely used to map the EMG signals captured at the amputated end to the bionic joints [23].Classification, a type of Supervised Machine Learning [24,25,26], was used to identify the hand movements corresponding to EMG signals recorded from a user. Geethanjali, P. (2016) used different types of classifiers to recognize patterns in the acquired EMG signals for operating a robotic hand, and the features and performances of the classifiers were determined [27].

This work aims to understand how a human hand functions by developing bionic models that mimic the movements of a human hand. In this work, two models are proposed to map the upper limb movements of a test person (collected from sensors) to corresponding angular displacements of bionic joints. The first model emphasizes finding an interpolated sensor value from consecutive sensor values using fuzzy logic. The second model uses a Regression-based pattern recognition model, by training an Artificial Neural Network (ANN)with recorded sensor data and testing it in real-time. It is concluded that the ANN model is better than the fuzzy logic model. Bionic elbow and wrist models were developed in the second model. The hand gestures of the test person were verified with movements of the bionic models. The human hand is considered as a rigid body here [23]. Only voluntary actions of the human's right hand are considered in this work.

${ }^{*}$ A test person is the one who has similar bone and muscle structure as that of the patient.

\section{DEVELOPMENT OF FUZZY LOGIC MODEL}

To capture the movements of elbow and wrist joints, a sensor glove was developed in which flex sensors [24] were placed at the joint portions of the glove. Flex sensors were used to capture flexion and extension actions of elbow and wrist joints. A rotary potentiometer was used to capture the turning of wrist. The list of joint movements and their ranges are tabulated in Table 1.

Table 1. Different movements of elbow and wrist.

\begin{tabular}{|c|c|c|c|}
\hline Joint & Movements & Minimum angle & Maximum angle \\
\hline Elbow & Flexion and extension & $30^{\circ}$ (flexion) $[25]$ & $180^{\circ}$ (extension) $[25]$ \\
\hline Wrist & Flexion and extension & $0^{\circ}$ (flexion) ${ }^{* 2}$ & $150^{\circ}$ (extension) ${ }^{* 3}$ \\
\hline Wrist & Turning & $0^{\circ}$ (supination) $[26]^{* 4}$ & $163^{\circ}{\text { (pronation) }[26]^{* 5}}^{*}$ \\
\hline
\end{tabular}


${ }^{*},{ }^{* 3}$ Note: While measuring the flexion angles of the test person's wrist, it was observed that the maximum flexion and extension occurred at $2^{\circ}$ and $148^{\circ}$ respectively (measured using Goniometers [27]). The angles were approximated to $0^{\circ}$ and $150^{\circ}$

${ }^{* 4},{ }^{* 5}$ Note: In the citation [26] the pronation and supination angles when the elbow is kept at $90^{\circ}$ are considered. Also, the values for pronation and supination given in the citation are $72.96^{\circ}$ and $88.59^{\circ}$ respectively. These angles are the same as $90^{\circ}+72.96^{\circ}=162.96^{\circ}$ and $90^{\circ}-88.59^{\circ}=0.41^{\circ}$ which are approximated to $163^{\circ}$ and $0^{\circ}$ respectively.

\subsection{Experimental Setup for the First Model}

The experimental setup for the first model is shown in Figure 1.

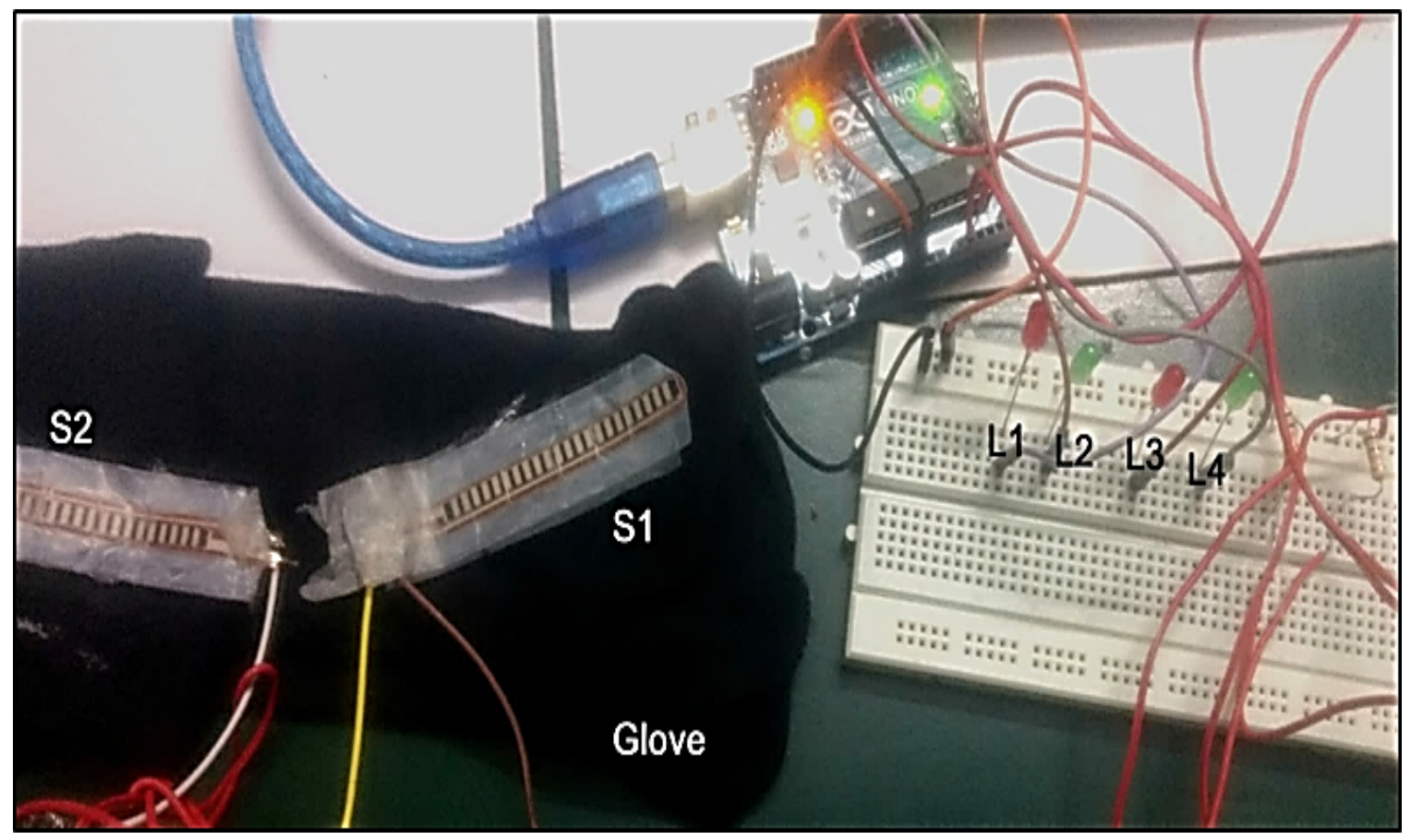

Figure 1. Experimental setup for measurement of flexion at elbow and wrist.

Flex sensors, $S 1$ and $S 2$ were attached to the elbow and wrist parts of a glove worn by the test person. LEDs $L 1, L 2, L 3$ and $L 4$ were used to indicate extreme elbow flexion, extreme elbow extension, extreme wrist flexion and extreme wrist extension, respectively. Flex sensor readings were taken continuously with an interval of $0.1 \mathrm{~s}$. Based on the difference between two consecutive readings, $\Delta S_{n}=\left|S_{n}-S_{n}-1\right|$ at time $t_{n}$, a fuzzy membership value $f m_{n}$ was assigned. An interpolated sensor value $p a_{n}$ was found using $f m_{n}$ and mapped to the range of joint's angular displacement values in a continuous range. Tables 2 and 3 show the assignment of fuzzy membership values to elbow and wrist flexion respectively. The steps involved in this model are shown in Figure 2. The assignment of fuzzy membership values for different values of the difference between consecutively recorded sensor values for elbow flexion and wrist flexion are listed in Tables 2 and 3 respectively. 
Table 2. Fuzzy membership value assignment $\left(f m_{n} l\right)$ based on the difference $\left(\Delta S_{n} 1\right)$ between consecutive flex sensor readings from $S 1$

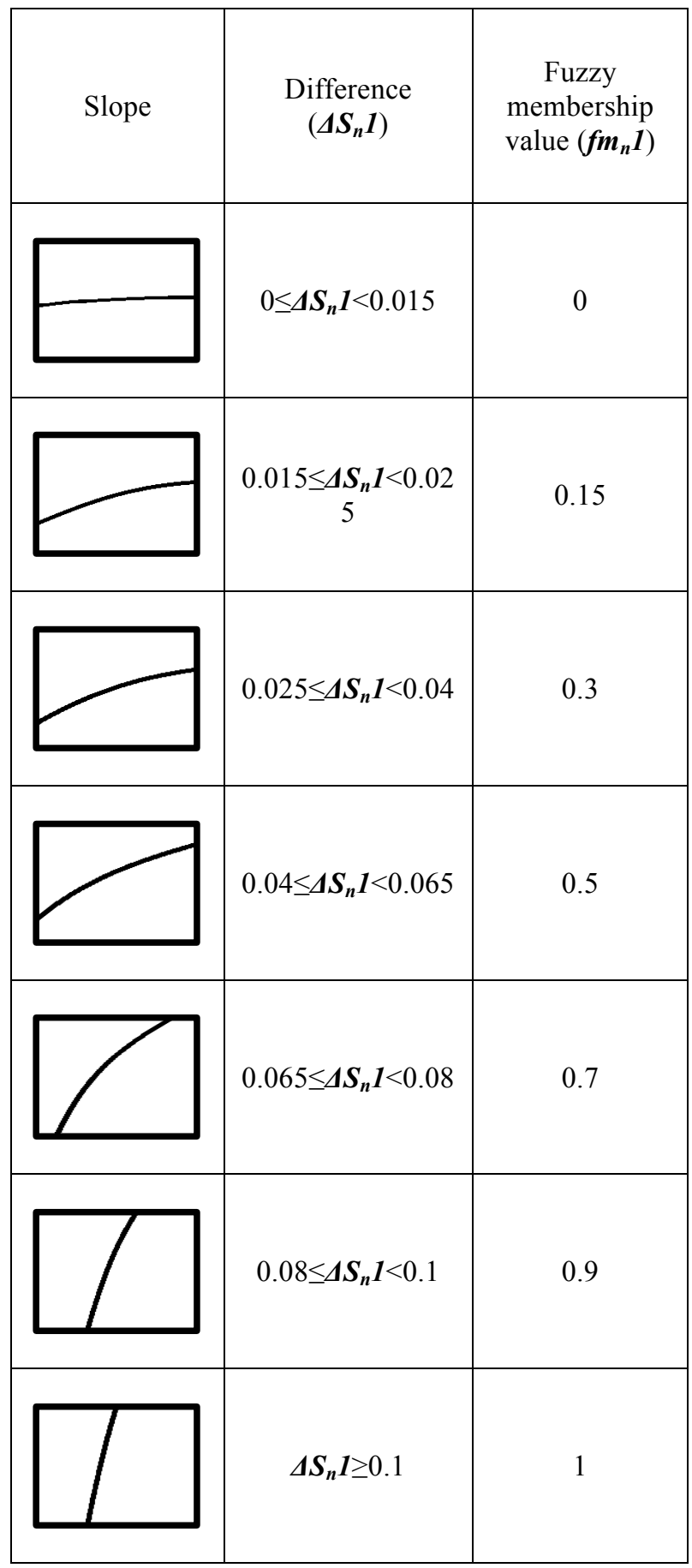

Table 3. Fuzzy membership value assignment $\left(f m_{n} 2\right)$ based on the difference $\left(\Delta S_{n} 2\right)$ between consecutive flex sensor readings from $S 2$

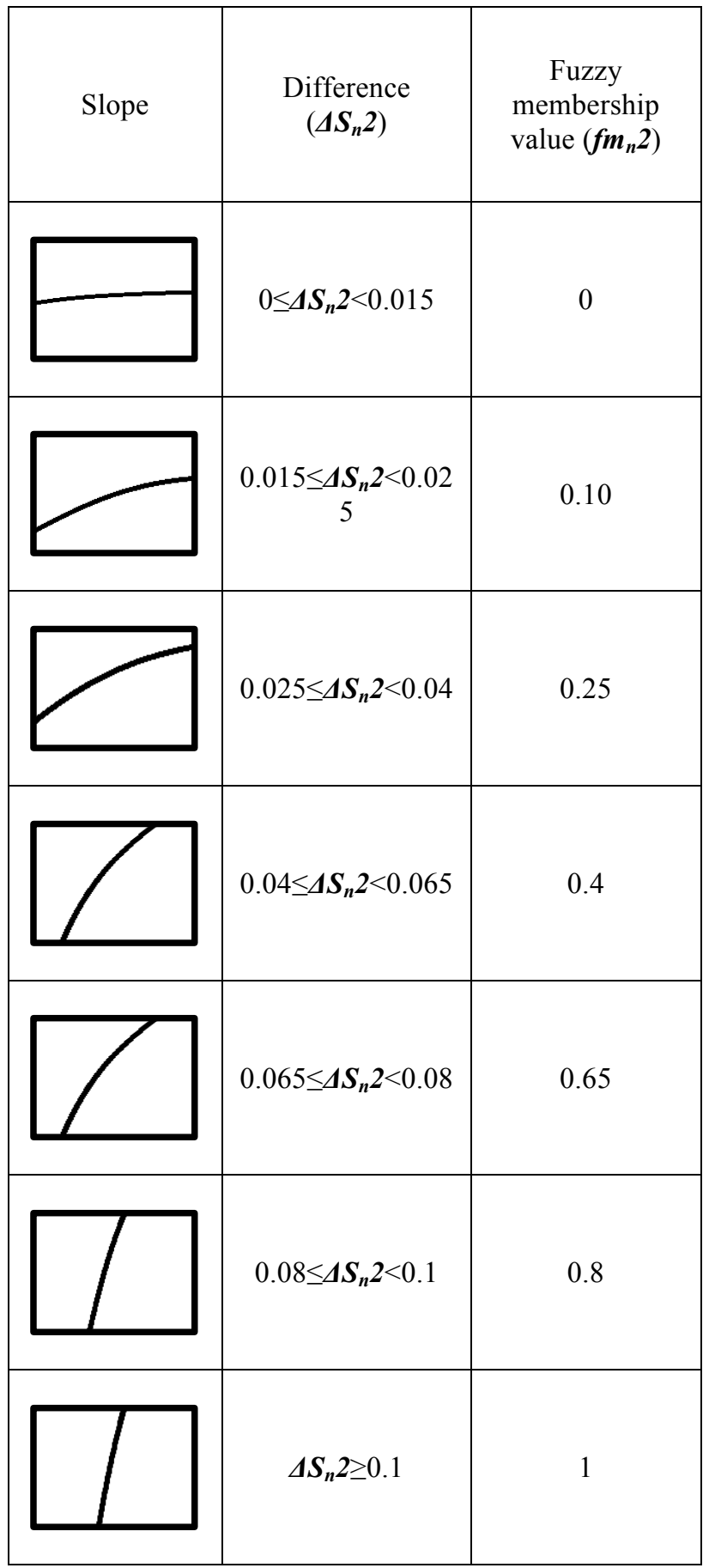




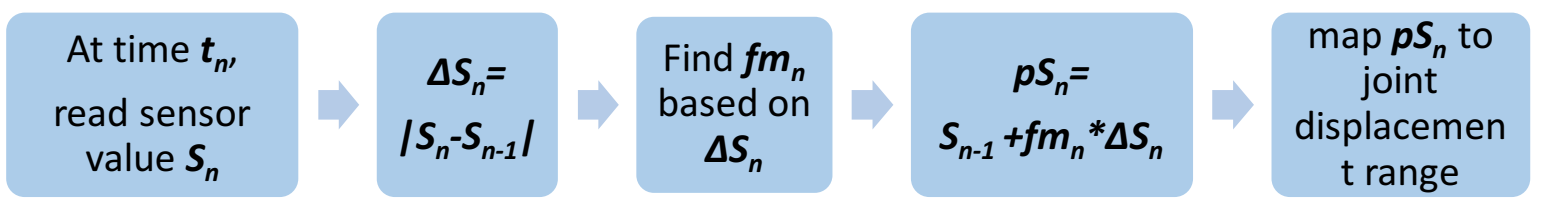

Figure 2. Control flow for Mapping using the fuzzy logic model.

Figures 3 and 4 show the plots of recorded sensor value versus time and the difference versus time, where, $e_{b}$ end $(t)$ and delta $e_{b} e n d(t)$ refers to flex sensor data at the elbow joint and the difference between consecutive sensor values, respectively. Figure 4 shows the flex sensor output and the difference between consecutive outputs at an interval of $0.1 \mathrm{~s}$.

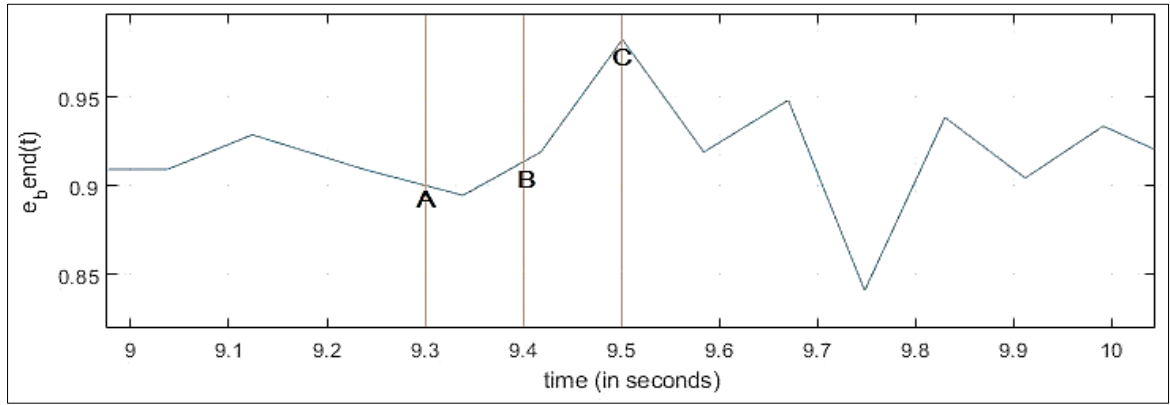

Figure 3. Output from flex sensor S1.

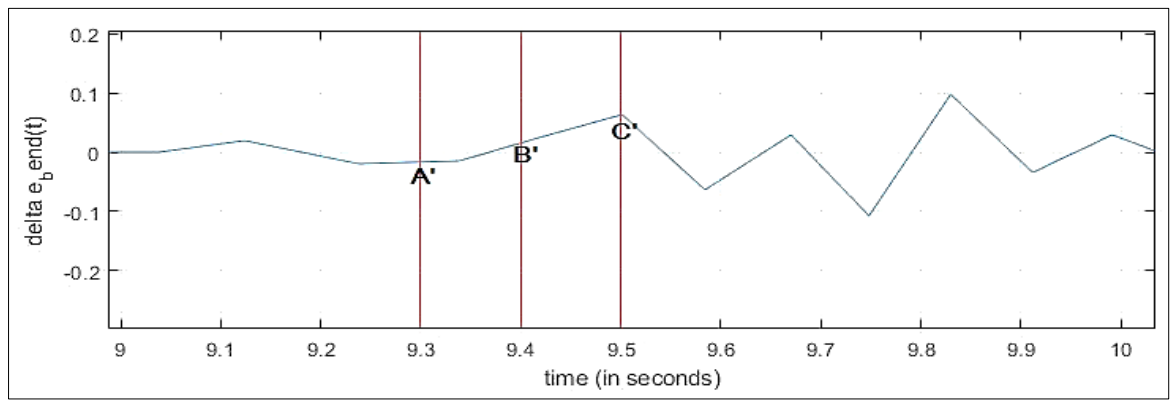

Figure 4. Difference between consecutive flex sensor (S1) readings.

From Figure 4, in the region A-B, there is a gradual change in flexion value, hence there is a smaller slope. In this region, the calculated interpolated sensor value has more membership in A than in $\mathrm{B}$. In the region B-C, the change in the flexion is quite rapid, hence the slope is steeper. In this case, the calculated interpolated sensor value has more membership in $\mathrm{C}$ than in $\mathrm{B}$.

Although the model was able to predict the angular displacement from the sensor values, it was observed that the model worked well for slow movements; it gave erroneous angles during rapid flexion and extension. The model could not cater to the overlapping of sensor values for neighboring categories, which resulted in mapping with considerable inaccuracies. 


\section{DEVELOPMENT OF NEURAL NETWORK-BASED MODEL}

In this model, the range of angular displacement values was discretized. For instance, flexion of wrist within the subrange $55^{\circ}$ to $64^{\circ}$ was categorized as $60^{\circ}$ (the target angle) and the subrange $65^{\circ}$ to $74^{\circ}$ was categorized as $70^{\circ} .16$ discrete categories for flexion were considered with an interval of $10^{\circ}$.

- $\quad$ Range of angles for elbow flexion: from $30^{\circ}$ (flexion) to $180^{\circ}$ (extension), Interval: $10^{\circ}$

- Range of angles for wrist flexion: from $0^{\circ}$ (flexion) to $150^{\circ}$ (extension), Interval: $10^{\circ}$

\subsection{Data Collection}

The sensor glove was worn by the test person. The elbow and wrist were positioned at different angles, which were verified using Goniometer (Figure 5).

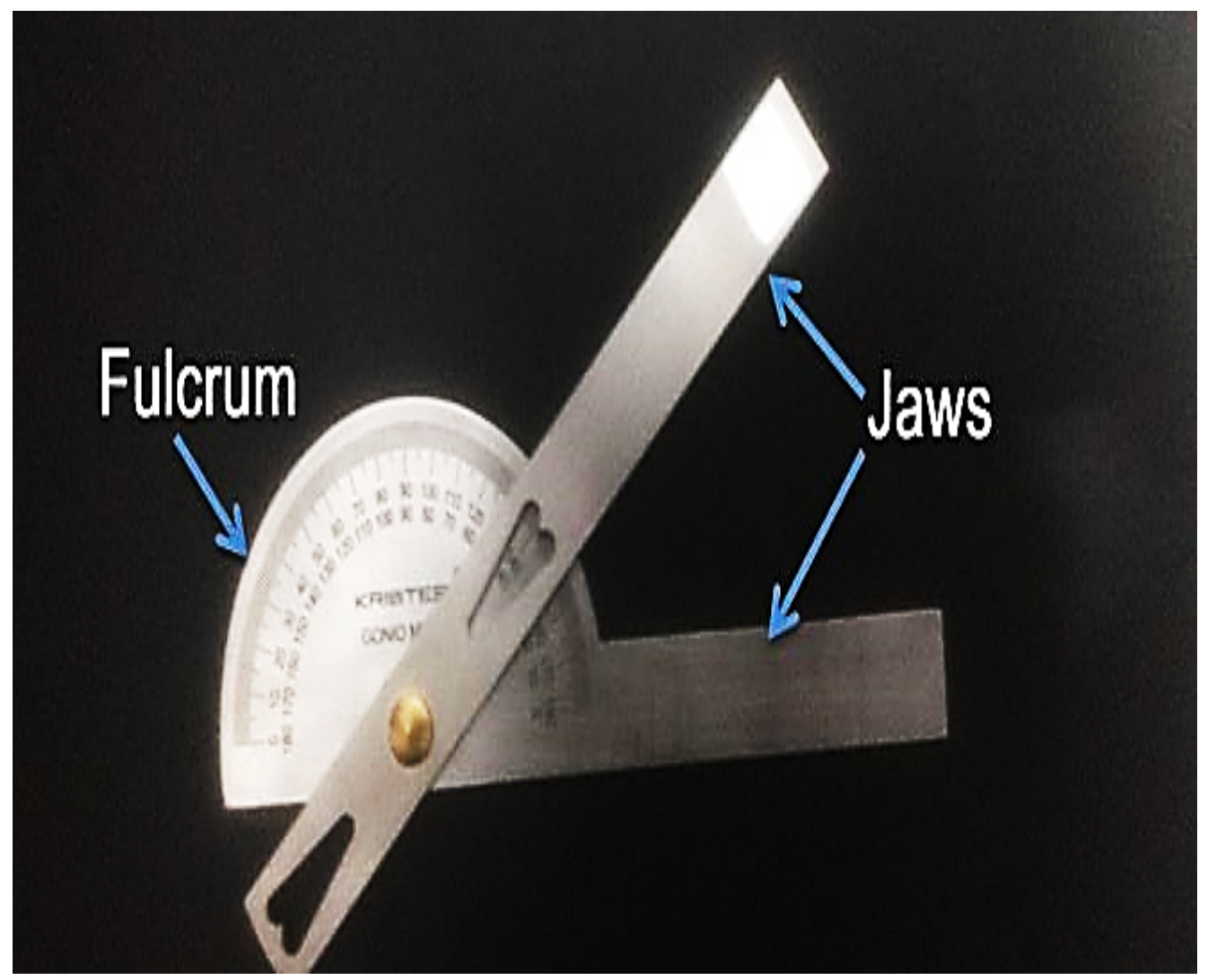

Figure 5. Goniometers.

Flex sensor readings for different angles in the range were taken. The overlapping of sensor values for an angle/category with that of the neighboring angles/categories ruled out the use of algorithms that involved linear interpolation. Figures 6 and 7 are the plots of flex sensor value versus time for different included angles at the elbow joint and wrist joint respectively. 


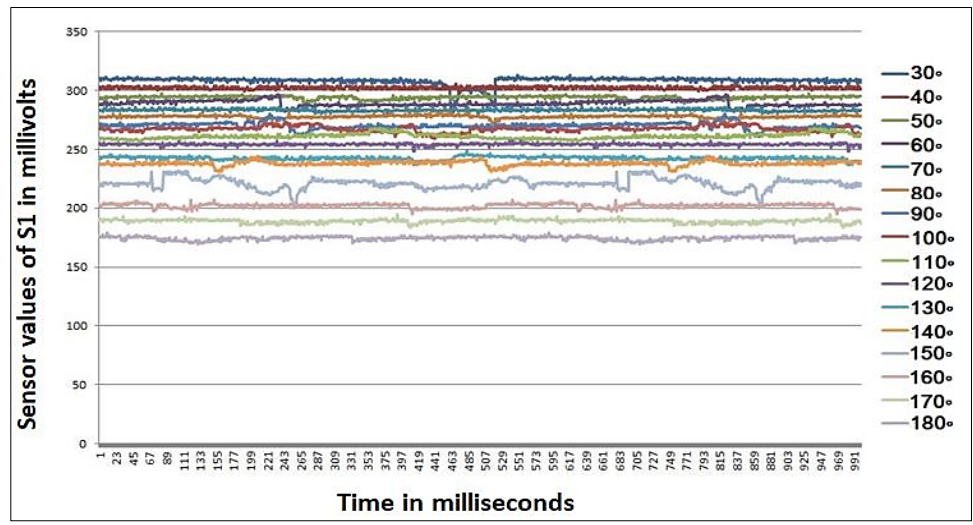

Figure 6. Sensor data collection from S1 for elbow flexion.

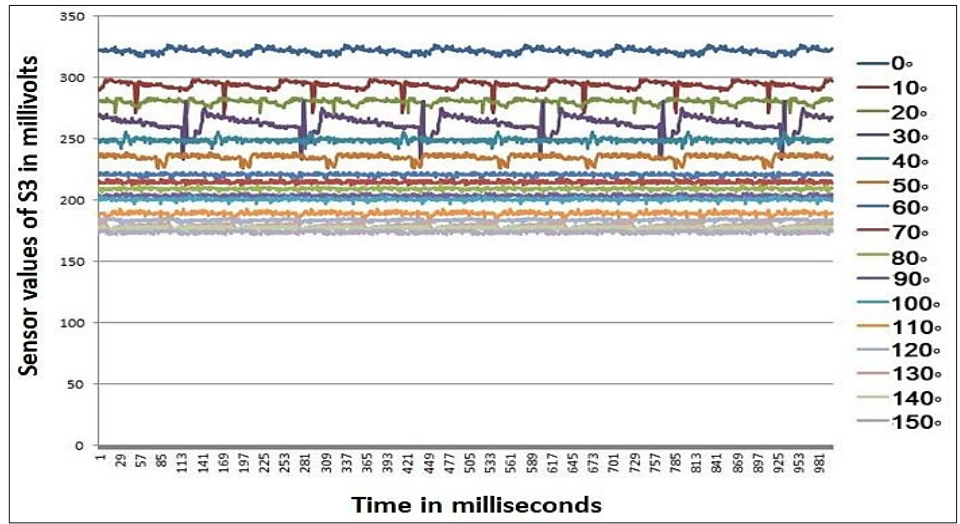

Figure 7. Sensor data collection from S3 for wrist flexion.

Since there was considerable overlapping between neighboring categories (angular displacements), the model was considered to be a regression problem under supervised machine learning. Mapping for rotation of wrist was also done in this model. For rotation of the wrist, 3 discrete states were considered: Supination $\left(0^{\circ}\right)$, Neutral position $\left(90^{\circ}\right)$ and Pronation $\left(163^{\circ}\right)$.

\subsection{Training and Optimization of the Neural Network}

A Neural Network was created using the Neural Network Toolbox in MATLAB and was trained with datasets to map a range of sensor values (input) to the required angular displacement of actuators (targets). The elbow was positioned at different angles from $30^{\circ}$ to $180^{\circ}$ with an interval of $10^{\circ}$. This was ensured using a goniometer [25].Sensor values from flex sensor S1 were recorded for each angle in the considered range. After numerous trials with a different number of datasets, different extents of overlapping and various parameters of the neural network, the network with maximum performance was taken into consideration. Thousand sensor values for one angle/category were considered; there were 16 datasets for 16 discrete angles $\left(30^{\circ}, 40^{\circ} \ldots\right.$ to $\left.180^{\circ}\right)$ and given as training data to the neural network. The neural network has 1 node in its input layer (input is taken from one sensor, S1), 2 hidden layers with 10 nodes and 20 nodes in it and 16 nodes in the output layer. The target datasets for the neural networks for elbow flexion and wrist flexion were prepared as shown in Figures 8 and 9 respectively. 


\begin{tabular}{|l|l|l|l|}
\hline \hline $30^{\circ}$ & $40^{\circ}$ & $50^{\circ}$ & $60^{\circ}$ \\
\hline 100000000000000 & 0100000000000000 & 0010000000000000 & 0001000000000000 \\
\hline \multicolumn{5}{|l|}{} \\
\hline $70^{\circ}$ & $80^{\circ}$ & $90^{\circ}$ & $100^{\circ}$ \\
\hline 0000100000000000 & 0000010000000000 & 0000001000000000 & 0000000100000000 \\
\hline \multicolumn{5}{|l|}{} \\
\hline $110^{\circ}$ & $120^{\circ}$ & $130^{\circ}$ & 140 \\
\hline 0000000010000000 & 0000000001000000 & 0000000000100000 & 0000000000010000 \\
\hline \multicolumn{5}{|l}{} \\
\hline $150^{\circ}$ & $160^{\circ}$ & $170^{\circ}$ & $180^{\circ}$ \\
\hline 0000000000001000 & 0000000000000100 & 0000000000000010 & 0000000000000001 \\
\hline
\end{tabular}

Figure 8. Targets for the neural network for elbow flexion.

\begin{tabular}{|c|c|c|c|}
\hline 0 & $10^{\circ}$ & 200 & $30^{\circ}$ \\
\hline 1000000000000000 & 0100000000000000 & 0010000000000000 & 0001000000000000 \\
\hline $40^{\circ}$ & $50^{\circ}$ & $600^{\circ}$ & $70^{\circ}$ \\
\hline 0000100000000000 & 0000010000000000 & 0000001000000000 & 0000000100000000 \\
\hline $80^{\circ}$ & $90^{\circ}$ & $100^{\circ}$ & $110^{\circ}$ \\
\hline 0000000010000000 & 0000000001000000 & 0000000000100000 & 0000000000010000 \\
\hline $120^{\circ}$ & $130^{\circ}$ & $140^{\circ}$ & $150^{\circ}$ \\
\hline 0000000000001000 & 0000000000000100 & 0000000000000010 & 0000000000000001 \\
\hline
\end{tabular}

Figure 9. Targets for the neural network for wrist flexion.

Note: The above steps are carried out for wrist flexion too, for range: $0^{\circ}$ to $150^{\circ}$ and an interval of $10^{\circ}(16$ targets), with the same specifications for its neural network.

The performance of the trained neural network depends on the number of hidden layers, number of nodes in the hidden layer(s), number of training data points given and degree of overlapping between neighboring categories. Different datasets were prepared for different degrees of categories' overlapping and tried in the network with different parameters.

The ANN was trained with the 1000 sensor values for each of the 16 categories of joint angles for elbow and wrist joints. The default features of the ANN are: 1000 input datapoints (flex sensor values) for each output category (joint angle), 2 hidden layers and 10 nodes and 20 nodes in the hidden layer. For different features of the ANN, the performance was noted in Table 4 and the corresponding ANN Training performance plots are shown in Figures 10, 11, and 12. The ANN Training performance plots are plots of the ANN's mean squared error (while training) versus the epoch, which is the number of passes for the ANN is passed with the datasets for training. It is a measure of the ANN's learning capacity.

Table 4. Performance of the ANN for different features (for elbow flexion data).

\begin{tabular}{|c|c|c|c|c|}
\hline S. No. & $\begin{array}{c}\text { Number of flex sensor } \\
\text { datapoints for each joint } \\
\text { angle category }\end{array}$ & $\begin{array}{c}\text { Number of } \\
\text { hidden } \\
\text { layer(s) }\end{array}$ & $\begin{array}{c}\text { Number of } \\
\text { nodes in the } \\
\text { hidden layer }\end{array}$ & $\begin{array}{c}\text { Performance of the } \\
\text { ANN (Accuracy in } \\
\text { mapping) }\end{array}$ \\
\hline 1 & 1000 & 1 & 10 & $91.875 \%$ \\
\hline
\end{tabular}




\begin{tabular}{|c|c|c|c|c|}
\hline 2 & 1000 & 1 & 100 & $91.458 \%$ \\
\hline $\begin{array}{c}3 \\
\text { (DEFAULT) }\end{array}$ & 1000 & 2 & 10,20 & $92.083 \%$ \\
\hline
\end{tabular}

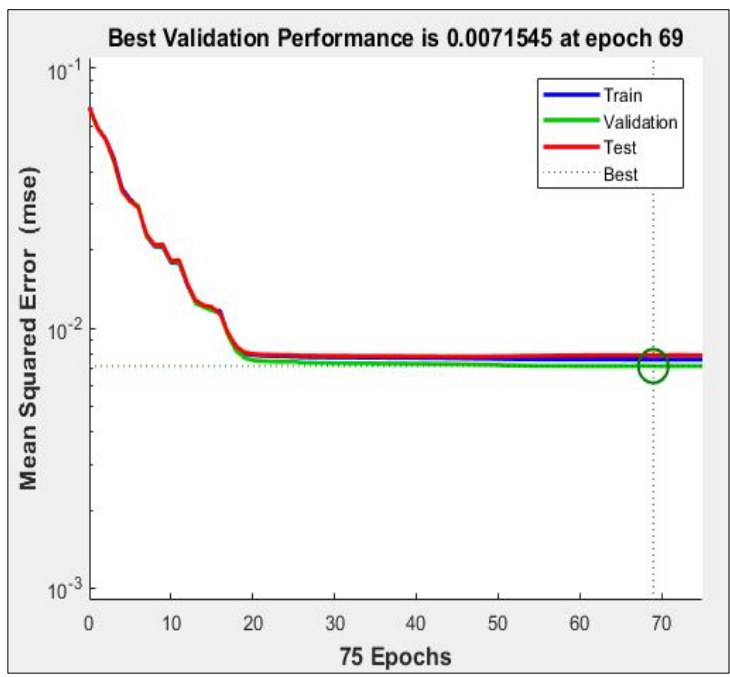

Figure 10. ANN performance for 1000 datapoints, 10 nodes in 1 hidden layer.

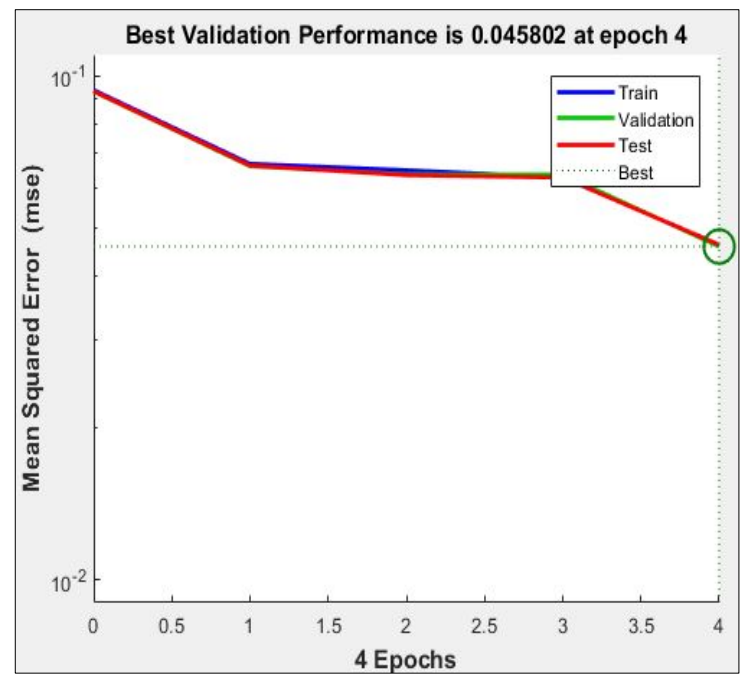

Figure 11. ANN performance for 1000 datapoints, 100 nodes in 1 hidden layer.

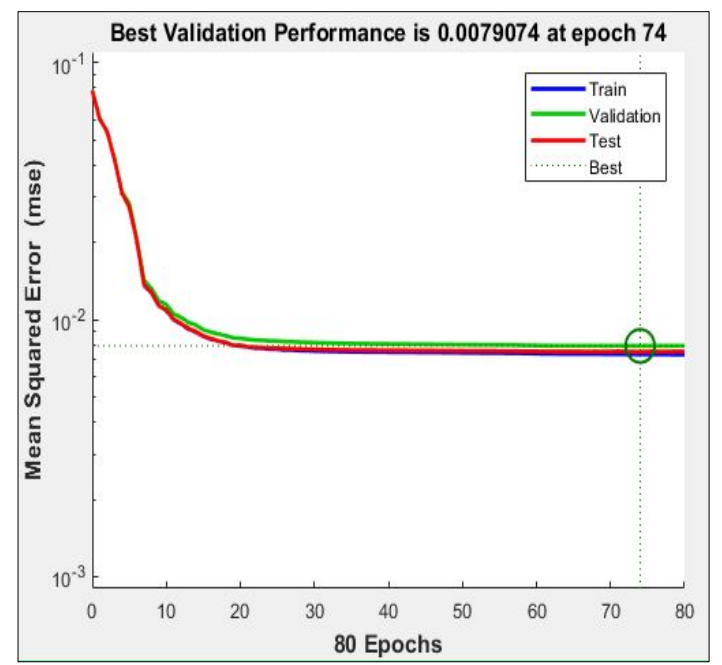

Figure 12. ANN performance for 1000 datapoints, 10, 20 nodes in 2 hidden layers. 
When the number of nodes in the hidden layer is increased, the performance of the ANN increases. However, if the number of hidden nodes is increased too far, then the ANN reaches a point of overfitting, where it learns the datapoints along with the noise which contributes to the lesser performance and higher mean squared error (Figure 11 compared to Figure 10). By increasing the number of hidden nodes, the ANN is trained with a lesser number of epochs, at the cost of computation time, which may cause delays while testing. Increasing the number of hidden layers increases the performance of the ANN as the hidden layer(s) are associated with the input layer that enables it to generate the desired outputs while training [28]. Increasing the number of input datapoints also increases the mapping performance of the ANN.

The maximum performances obtained for elbow flexion and wrist flexion are $92.083 \%$ (Figure 13) and 94.458\% (Figure 14), respectively.

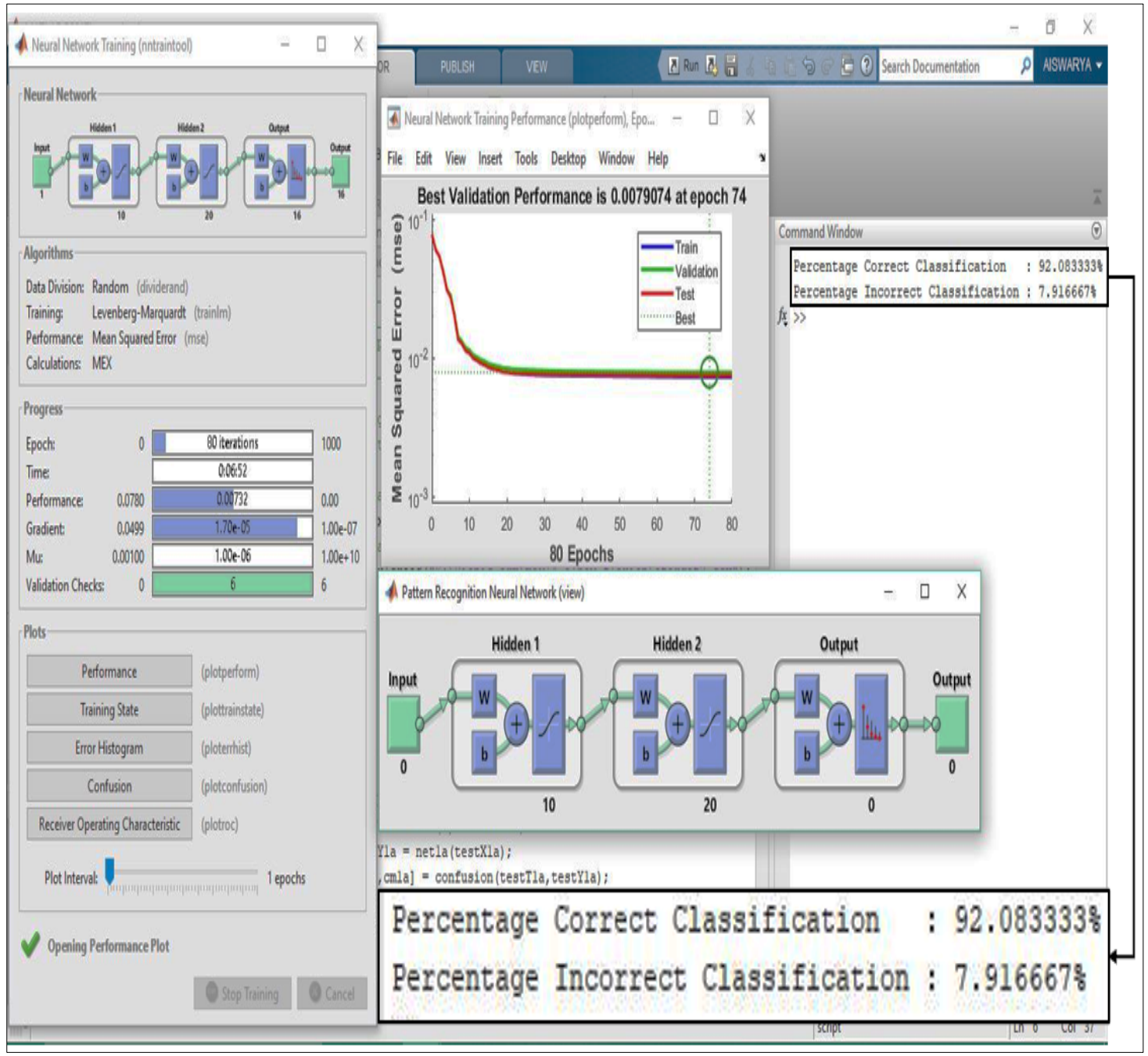

Figure 13. Testing the ANN for elbow flexion. 


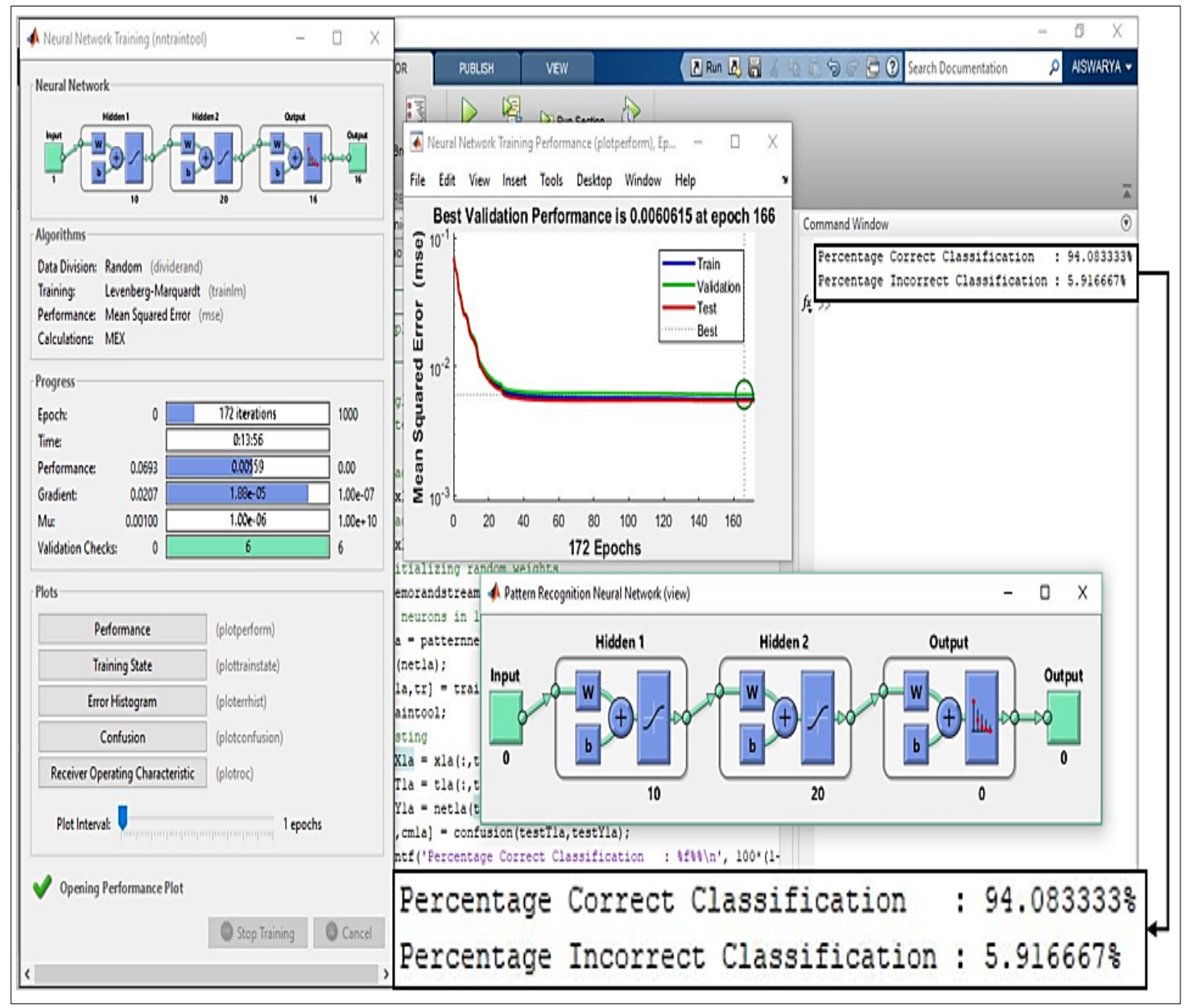

Figure 14. Testing the ANN for wrist flexion.

\subsection{Implementation of NN Models Into Bionic Models of Elbow And Wrist}

Mechanical models of the Bionic arm, with a scaling factor of 1:1, have been made using Sunboard and integrated with the circuit (Figure 15). The models were designed to replicate the actions of a human hand. Servo motors M1, M2 and M3 were used to actuate the joint motors for elbow flexion, wrist rotation and wrist flexion respectively. Separate models for bionic elbow and bionic wrist were done due to load carrying constraints of servo motor M1. Flex sensors S1 and S3 were used to capture flexion of elbow and wrist respectively [29]. To capture the rotation of the wrist, a rotatory potentiometer, S2, which is very similar to haptic knob (used for rehabilitation of hand functionality), was used [30]. LEDs LA, LB and LC were used to indicate the pronation, neutral position and supination positions of a wrist respectively [31]. Two neural networks were created and trained with datasets of flex sensor values for each angle. They were tested with real-time data from sensors S1 and S3 (due to elbow flexion and wrist flexion test person who wore the flex glove). The outputs of the tested neural network were angular displacement values of the elbow joint and wrist joint, were written onto servo motors M1 and M3 in the bionic arm models (Figure 15). For rotation of the wrist, sensor values from S2 were recorded, based on which servo motor M2 was controlled (Figure 16). 


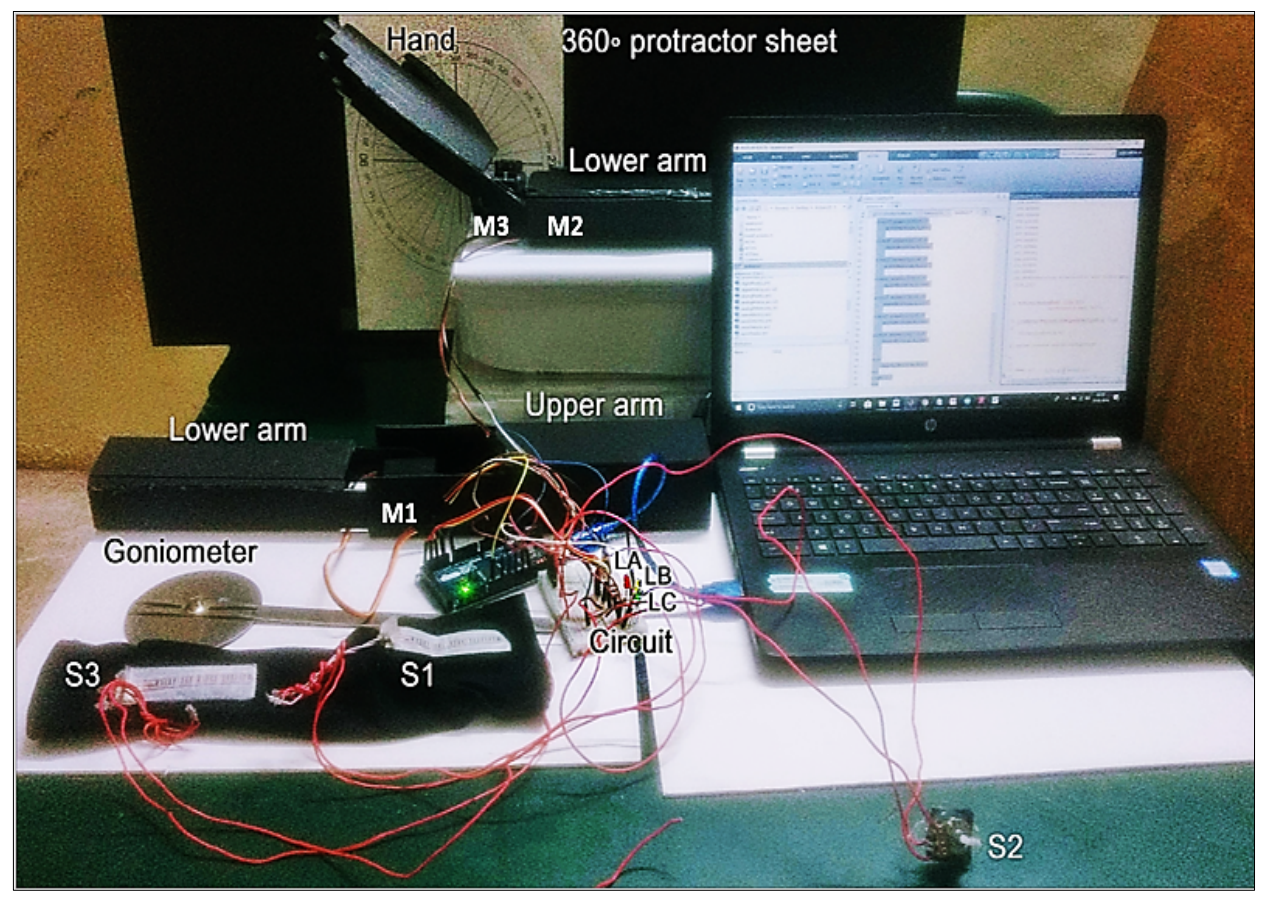

Figure 15. Experimental setup of the ANN based model.

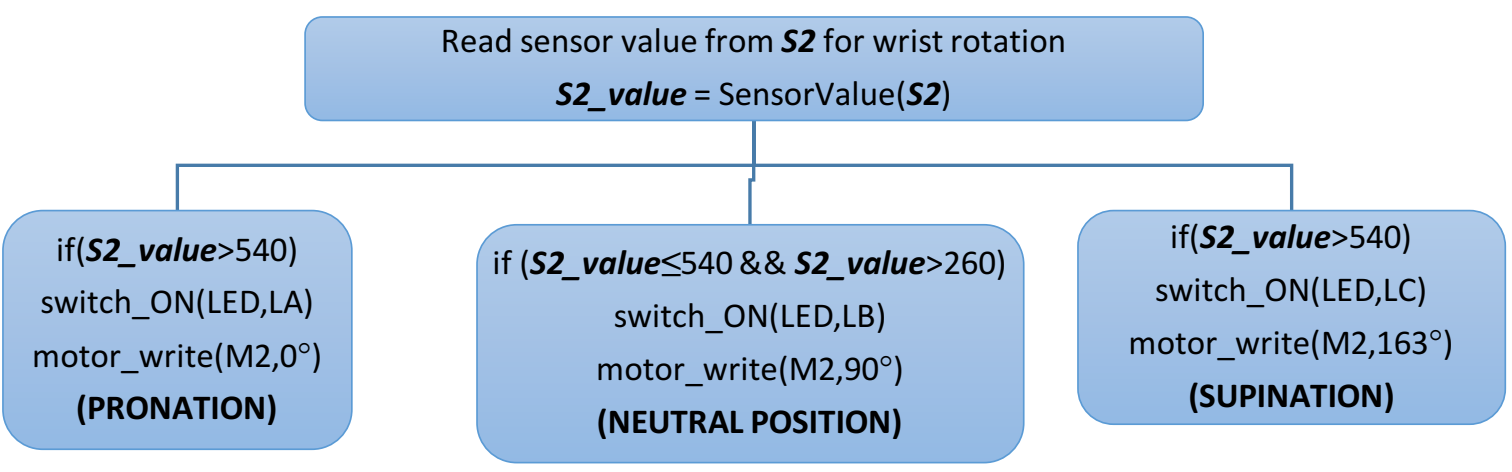

Figure 16. Control flow for rotation of bionic wrist joint.

This model was verified in real-time using goniometers to measure the flexion angles at the joints of the test person and using a $360^{\circ}$ protractor sheet to measure the flexion angles at the joints of the bionic models.

\section{RESULTS OF THE WORK}

In Model 1, the flexion and extension of the elbow and wrist were captured using flex sensors. The maximum flexion and extension were indicated using the corresponding LEDs. The logic involved mapping the sensor values to a continuous range of angles. Extreme cases of wrist flexion are shown in Figure 17. 


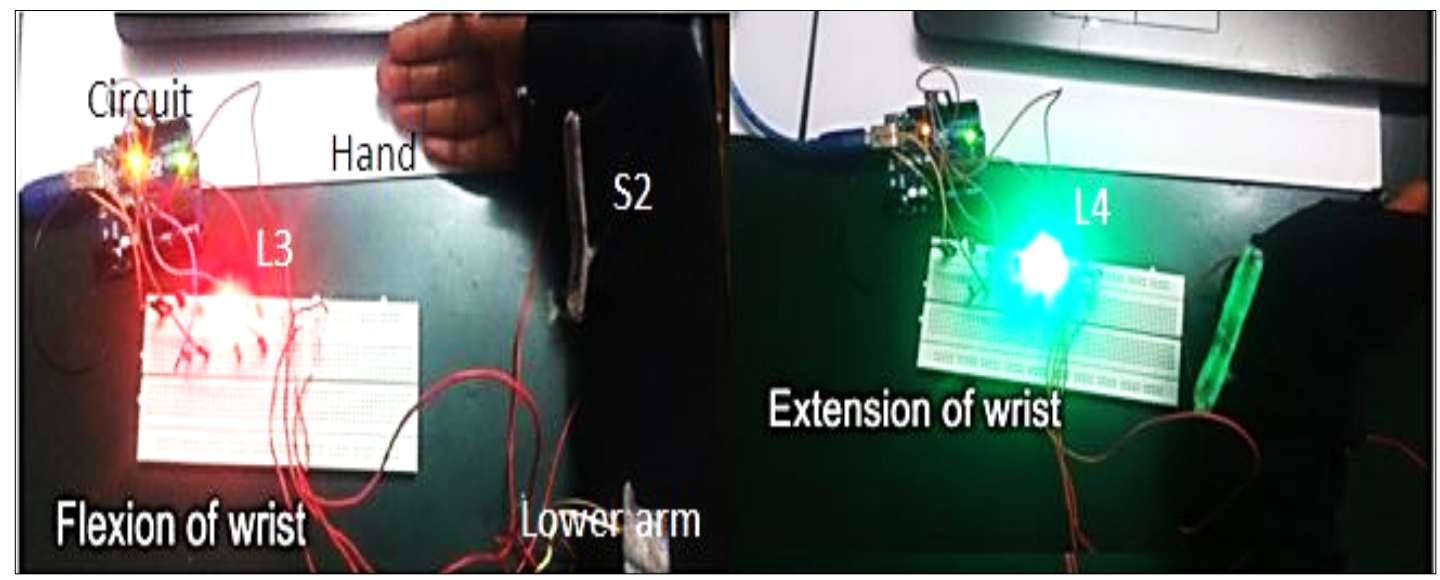

Figure 17. Flexion and Extension of the wrist (Model 1).

The first model focused on finding an interpolated value between the previously measured value and the currently measured value using fuzzy logic and mapping that value to the actuation of the corresponding joint motor using linear interpolation. The model worked well in the case of extreme flexion and extreme extension for the elbow and wrist joints and for slower transitions while flexing. The model was not able to map rapid changes from the flex sensor, thus leading to inaccurate joint motor values. In Model 2, flexion and extension of elbow and wrist were captured using flex sensors for 16 different angles' categories. Additionally, rotation of the wrist was captured using a rotary potentiometer for pronation, supination and neutral position of the wrist. A neural network mapping was done to map the flexion of the elbow and wrist to their corresponding angular displacement values. The range consists of 16 different angle ranges for both the cases of flexion and 3 different angles for rotation of the wrist. Mechanical models of the bionic elbow and bionic wrist were constructed with motors attached to the joints to simulate the hand movements of the test person. Extension and flexion of the elbow in Model 2 are shown in Figures 18 and 19. For the wrist joint, extreme flexion and flexion at $90^{\circ}$ in Model 2 are shown in Figures 20 and 21, respectively.

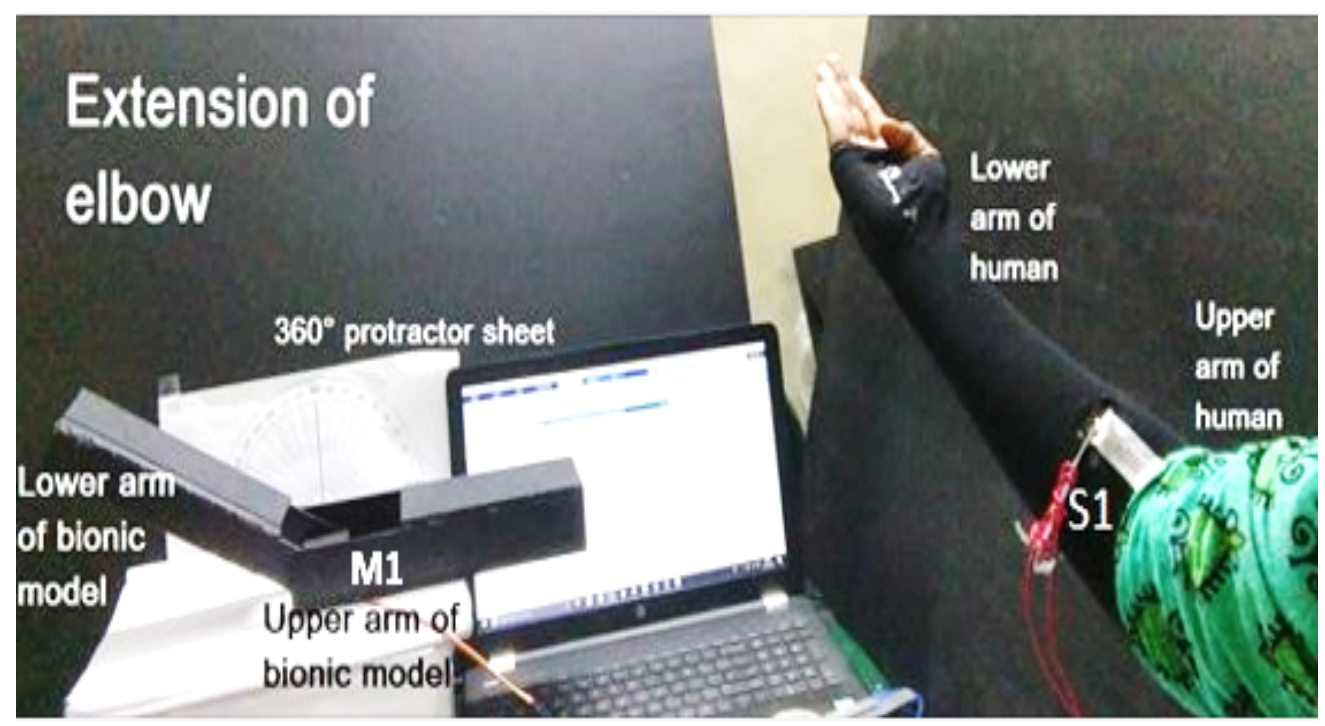

Figure 18. Mapping of elbow extension. 


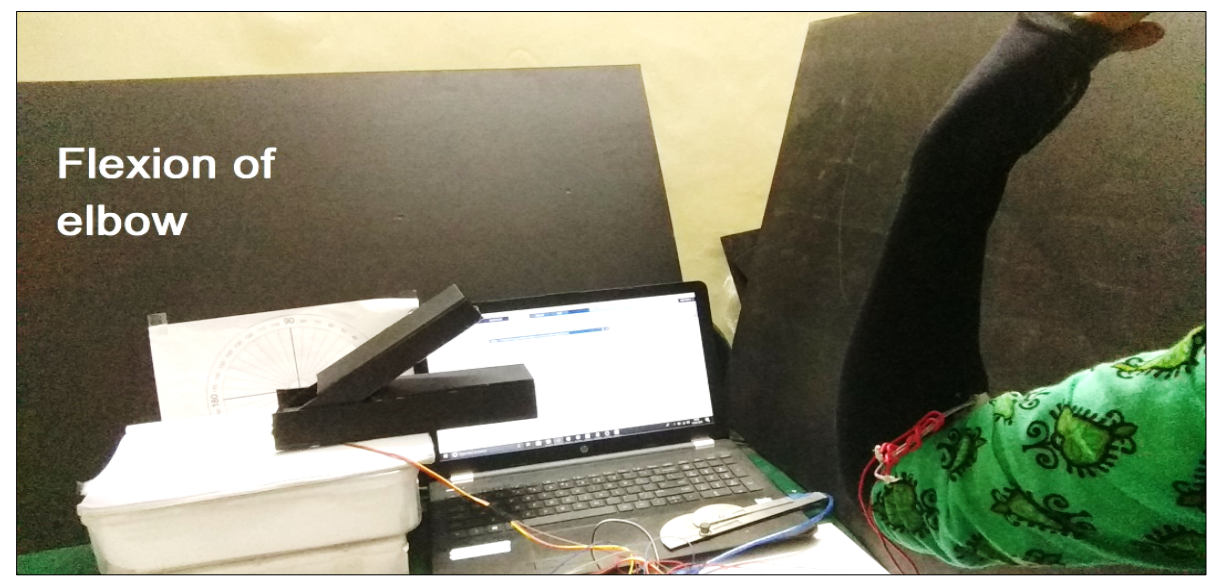

Figure 19. Mapping of elbow flexion.

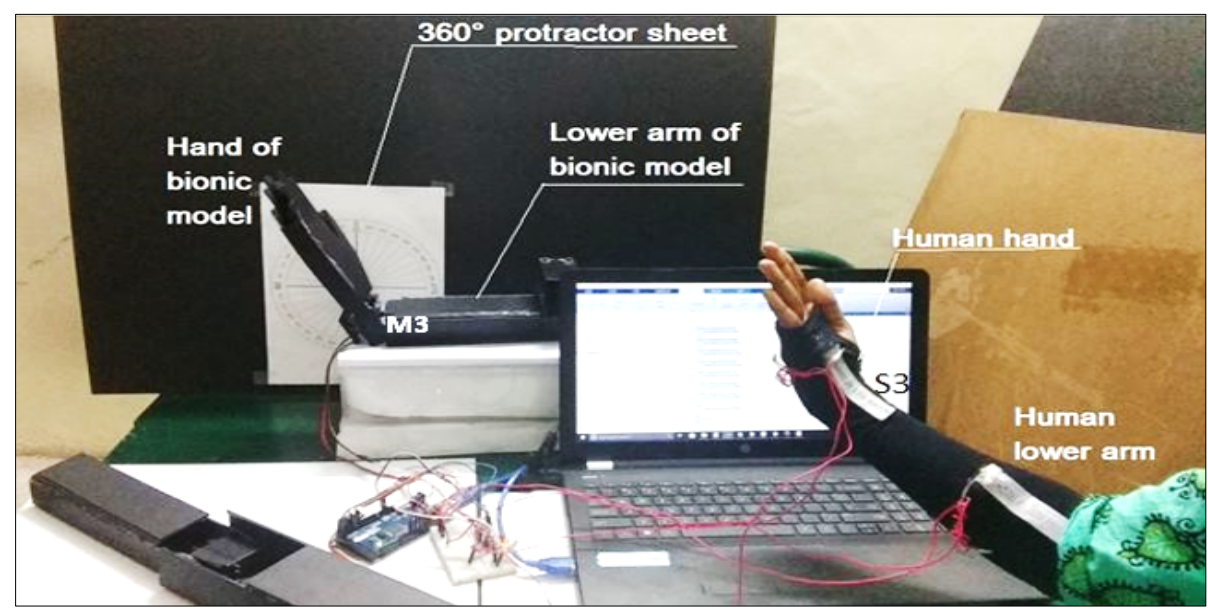

Figure 20. Mapping of wrist flexion.

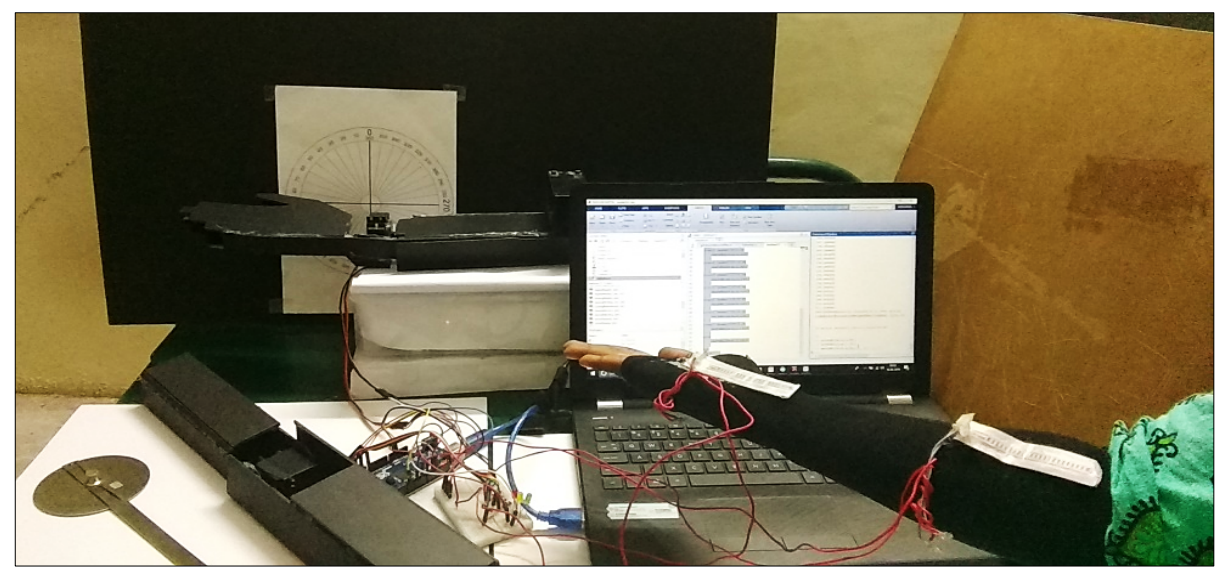

Figure 21. Mapping of wrist flexion (at $90^{\circ}$ ). 
Wrist rotation was done similar to a haptic knob to capture and map wrist pronation, neutral position, and supination

(Figure 22).

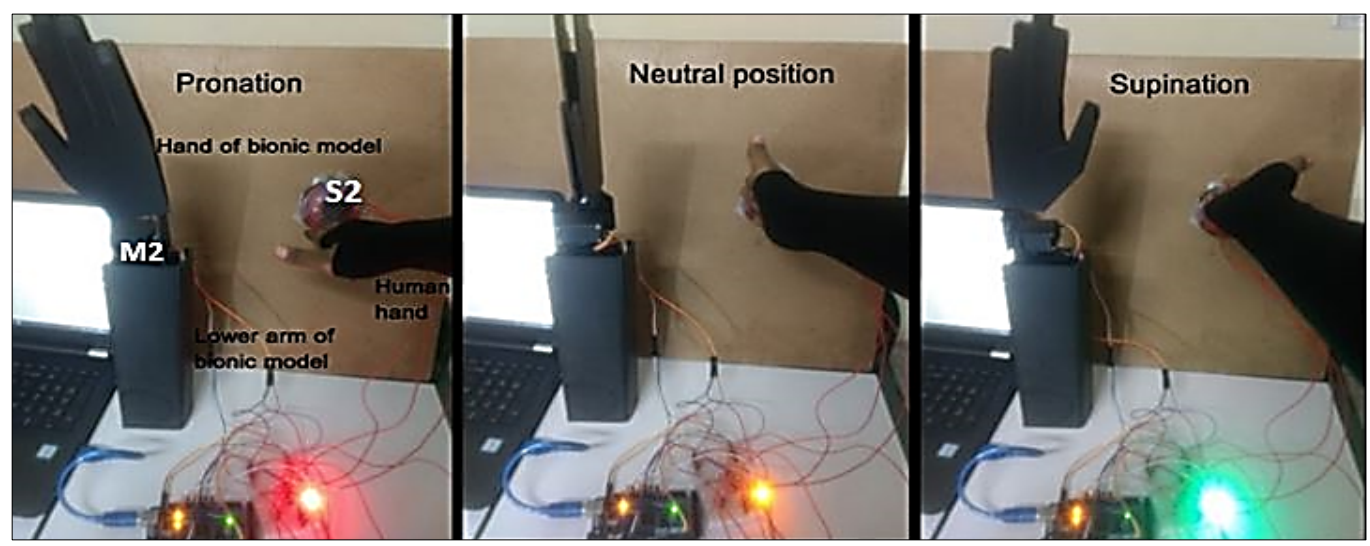

Figure 22. Mapping of pronation, neutral position and supination of the wrist.

The first model could not be verified as the output range was continuous and it was difficult to measure the angle included at the bionic joints. The verification of the second model is given in Table 5, in which the number of success trials refers to the number of testing trials when the angle made by the test person's joint (measured using Goniometer) coincided with the angle made at the corresponding bionic joint (measured using the $360^{\circ}$ protractor sheet).

Table 5. Verification of Model 2.

\begin{tabular}{|c|c|c|c|}
\hline Joint & Elbow flexion & Wrist rotation & Wrist flexion \\
\hline Sensor & Flex sensor & Rotary potentiometer & Flex sensor \\
\hline Mapping Method & ANN & Conditional clause & ANN \\
\hline Output range & $\left\{30^{\circ}, 40^{\circ}, \ldots 180^{\circ}\right\}$ & $\left\{0^{\circ}, 90^{\circ}, 163^{\circ}\right\}$ & $\left\{0^{\circ}, 10^{\circ}, \ldots 150^{\circ}\right\}$ \\
\hline Number of testing trials $n(T)$ & $\begin{array}{c}48 \text { ( } 3 \text { trials for } \\
\text { each angle) }\end{array}$ & $\begin{array}{c}15 \text { (5 trials for each } \\
\text { angle) }\end{array}$ & $\begin{array}{c}48 \text { (3 trials for each } \\
\text { angle) }\end{array}$ \\
\hline Number of success trials $n(S)$ & 40 & 15 & 42 \\
\hline $\begin{array}{c}\text { Experimental performance } \\
=\frac{n(S)}{n(T)} * \mathbf{1 0 0}(\%)\end{array}$ & $83.33 \%$ & $100 \%$ & $87.5 \%$ \\
\hline Estimated ANN Performance & $92.083 \%$ & ANN was not used & $94.083 \%$ \\
\hline
\end{tabular}




\section{CONCLUSION}

To understand the development and control of a Transhumeral bionic, 2 models were developed in this work to map the flexion of elbow and wrist to the corresponding angular displacements of joints in a bionic arm model. A flex glove was developed (flex sensors placed at the elbow and wrist joints), worn by the test person, and sensor readings were taken depending on the flexion movements of the test person.

The first model was based on fuzzy logic. Based on the difference in two consecutive flex sensor readings $\left(S_{n-}\right.$ ${ }_{1}, S_{n}$ ), an interpolated sensor value was determined, that had fuzzy membership in $S_{n-1}$ and $S_{n}$. The fuzzy membership value was determined using the difference between $S_{n-1}$ and $S_{n}$. Lesser the difference, more the membership of the interpolated sensor value in $S_{n-1}$ and vice-versa. The interpolated flex sensor value was mapped to the angular displacement of the joint using linear interpolation. However, the model didn't account rapid flexion changes and was inaccurate as the domain (flex sensor range) and range (servo motor range) were continuous.

In the second model, the range of angular displacements was categorized into 16 discrete sets of angles for flexion of elbow (from $30^{\circ}$ to $180^{\circ}$ ) and wrist (from $0^{\circ}$ to $150^{\circ}$ ). To address the overlapping problem encountered in the previous model, a machine learning approach was sought. A neural network was created in MATLAB to map the flex sensor value to the corresponding joint's angular displacement. For each of the 16 angles' categories, flex sensor values were recorded for the input datasets. The neural network was trained using the input datasets and tested. This method was carried out for flexion at elbow and wrist joint. The rotation at the wrist joint was captured using a rotary potentiometer, which is similar to a haptic knob. Bionic models of elbow and wrist were fabricated with servo motors placed at their joints. While testing in real-time, the bionic models actuated in the way the hand of the test person moved.

\section{INFERENCES}

- In this work, a fuzzy-based mapping model was compared to Supervised Machine Learning-based pattern recognition Artificial Neural Networks (ANN) model. It discusses the effectiveness and accuracy of the latter model over the former for mapping sensory signals in Bionics.

- It has given the idea that sensors used in bionics are prone to give overlapping values for different actuations of flexion. The problem could be encountered by dividing the sensor range to sub-ranges and discretizing the actuation range. Mapping using ANNs give better results as the mapping will be done from a known range of values.

- It is also essential to carry out the experiment of collecting muscle flexion data with a test person before actually implementing on an amputated person.

\section{REFERENCES}

SR ElKoura, G. \& Singh, K. 2003. Handrix: Animating the human hand. Proceedings of the 2003 ACM SIGGRAPH/Eurographics Symposium on Computer Animation: 110-119.

Mio, R., Villegas, B., Ccorimanya, L., Flores, K.M., Salazar, G., \& Elías, D. 2017. Development and assessment of a powered 3D-printed prosthetic hand for transmetacarpal amputees. 2017 3rd International Conference on Control, Automation and Robotics (ICCAR), IEEE: 85-90.

SSalminger, S., Vujaklija, I., Sturma, A., Hasenoehrl, T., Roche, A.D., Mayer, J.A., Hruby, L.A. \&Aszmann, O.C. 2019. Functional outcome scores with standard myoelectric prostheses in below-elbow amputees. American journal of physical medicine \& rehabilitation 98(2): 125-129. 
Betsy Dayana Marcela Chaparro-Rico, Daniele Cafolla, Eduardo Castillo-Castaneda, and Marco Ceccarelli. 2020," Design of arm exercises for rehabilitation assistance", Journal of Engineering Research, Vol 8(3):203-218

Cumming, J., Barr, S. \& Howe, T.E. 2006. Prosthetic rehabilitation for older dysvascular people following a unilateral transfemoral amputation. Cochrane Database of Systematic Reviews (4).

Chen, W., Xiong, C., \& Yue, S. 2015. On configuration trajectory formation in spatiotemporal profile for reproducing human hand reaching movement. IEEE Transactions on Cybernetics 46(3): 804-816.

Shen, S. S., Lin, S. H., Kang, T. H., \& Chien, W. 2016. Enhanced keystroke dynamics authentication utilizing pressure detection. 2016 International Conference on Applied System Innovation (ICASI): 1-4. IEEE.

Farina, D., \&Amsüss, S. 2016. Reflections on the present and future of upper limb prostheses. Expert review of medical devices 13(4): 321-324.

Parithimarkalaignan, S., \& Padmanabhan, T. V. 2013. Osseointegration: an update. The Journal of Indian Prosthodontic Society 13(1): 2-6.

Kuiken, T. A., Barlow, A. K., Hargrove, L., \&Dumanian, G. A. 2017. Targeted muscle reinnervation for the upper and lower extremity. Techniques in orthopaedics (Rockville, Md.) 32(2): 109.

Mioton, L. M., \&Dumanian, G. A. 2018. Targeted muscle reinnervation and prosthetic rehabilitation after limb loss. Journal of Surgical Oncology 118(5): 807-814.

Hargrove, L. J., Miller, L. A., Turner, K., \&Kuiken, T. A. 2017. Myoelectric pattern recognition outperforms direct control for transhumeral amputees with targeted muscle reinnervation: a randomized clinical trial. Scientific reports 7(1): 1-9.

Resnik, L., Cancio, J., Fantini, C., Ikeda, A., \&Sasson, N. 2017. Pattern recognition control of the DEKA arm in two transhumeral amputees with targeted muscle reinnervation. Proceeding of MEC 17.

Barone, R., Ciancio, A. L., Romeo, R. A., Davalli, A., Sacchetti, R., Guglielmelli, E., \&Zollo, L. 2016. Multilevel control of an anthropomorphic prosthetic hand for grasp and slip prevention. Advances in Mechanical Engineering 8(9): 1687814016665082.

Mangukiya, Y., Purohit, B., \& George, K. 2017. Electromyography (EMG) sensor controlled assistive orthotic robotic arm for forearm movement. 2017 IEEE Sensors Applications Symposium (SAS): 1-4. IEEE.

Gauthaam, M., \& Kumar, S. S. 2011. EMG controlled bionic arm. 2011 National Conference on Innovations in Emerging Technology: 111-114. IEEE.

Zhuojun, X., Yantao, T., \& Yang, L. 2015. sEMG pattern recognition of muscle force of upper arm for intelligent bionic limb control. Journal of Bionic Engineering 12(2): 316-323.

Chen, W., Xiong, C., \& Yue, S. 2015. On configuration trajectory formation in spatiotemporal profile for reproducing human hand reaching movement. IEEE Transactions on Cybernetics 46(3): 804-816.

Chen, W., Xiong, C., \& Yue, S. 2015. On configuration trajectory formation in spatiotemporal profile for reproducing human hand reaching movement. IEEE Transactions on Cybernetics 46(3): 804-816.

Taşar, B., \&Gülten, A. 2017. EMG-Controlled Prosthetic Hand with Fuzzy Logic Classification Algorithm. Modern Fuzzy Control Systems and Its Applications, 321.

Muhammad Shahzad, 2020, "Fuzzy Logic Based Prediction Algorithm Using Nearest Neighborhood Clustering, Journal of engineering research, Vol 8 (3): 135-152.

Meisam Nasrollahi, Jafar Razmi, Reza Ghodsi. 2019, “A combined fuzzy PCA approach for location optimization and capacity planning in Glycyrrhizae green supply network design", Journal of engineering research, Vol 7 (4):261-275 
Xu, K., Guo, W., Hua, L., Sheng, X., \& Zhu, X. 2016. A prosthetic arm based on EMG pattern recognition. 2016 IEEE International Conference on Robotics and Biomimetics (ROBIO): 1179-1184. IEEE.

Sharmila, K., Sarath, T. V., \& Ramachandran, K. I. 2016. EMG controlled low cost prosthetic arm. 2016 IEEE Distributed Computing, VLSI, Electrical Circuits and Robotics (DISCOVER): 169-172. IEEE.

Hima Bindu, Manjunathachary K. 2019, "Kernel-based scale-invariant feature transform and spherical SVM classifier for face recognition", Journal of engineering research, Vol 7(3):142-160.

Abdullah Alqallaf 2019, "Focused Synthetic Radar Imaging Approach for Multi-tumors Screening", Journal of engineering research, Vol 7(3): 232-243.

Geethanjali, P. 2016. A mechatronics platform to study prosthetic hand control using EMG signals. Australasian physical \& engineering sciences in medicine 39(3), 765-771.

Shen, S. S., Lin, S. H., Kang, T. H., \&Chien, W. 2016. Enhanced keystroke dynamics authentication utilizing pressure detection. In 2016 International Conference on Applied System Innovation (ICASI): 1-4. IEEE.

Saggio, G., Riillo, F., Sbernini, L., \&Quitadamo, L. R. 2015. Resistive flex sensors: a survey. Smart Materials and Structures 25(1): 013001.

Resende, T., Carneiro, A. A. L., Moretti, E., Boaviagem, A., dos Santos Cardozo, L. B., Moreira, M. C., \& Caldas, R. 2017. Universal goniometer and smartphone app for evaluation of elbow joint motion: reproducibility analysis. 2017 International Conference on Virtual Rehabilitation (ICVR): 1-2. IEEE.

Vetrice, G., \&Deaconescu, A. 2017. Development of elbow rehabilitation equipment using pneumatic muscles. MATEC Web of Conferences (Vol. 121, p. 01017). EDP Sciences.

Rahman, H. A., Fai, Y. C., \& Ming, E. S. L. 2014. Analysis of human hand kinematics: Forearm pronation and supination. Journal of Medical Imaging and Health Informatics 4(2): 245-249.

Freeman, J. A., \&Skapura, D. M. 1991. Neural networks: algorithms, applications, and programming techniques. Addison Wesley Longman Publishing Co., Inc. 89-102.

Malagelada, F., Dalmau-Pastor, M., Vega, J., \&Golano, P. 2014. Elbow anatomy. Sports injuries: prevention, diagnosis, treatment and rehabilitation, 2: 527-53.

Lambercy, O., Dovat, L., Yun. H., Wee. S. K., Kwah. C. W., Chua. K. S., Gassert. R., Milner, T. E., Teo, C. L., \&Burdet. E. 2011. Effects of a robot-assisted training of grasp and pronation/supination in chronic stroke: a pilot study. Journal of NeuroEnginerring and Rehabilitation, 8(63).

Kane, P. M., Vopat, B. G., Got, C. J., Mansuripur, K., \&Akelman, E. 2014. The Effect of Supination and Pronation on Wrist Range of Motion. Journal of wrist surgery 3(3): 187-91. 